\title{
Processors for ALOS Optical Data: Deconvolution, DEM Generation, Orthorectification, and Atmospheric Correction
}

\author{
Peter Schwind, Mathias Schneider, Gintautas Palubinskas, Tobias Storch, Rupert Müller, and Rudolf Richter
}

\begin{abstract}
The German Aerospace Center (DLR) is responsible for the development of prototype processors for PRISM and AVNIR-2 data under a contract of the European Space Agency. The PRISM processor comprises the radiometric correction, an optional deconvolution to improve image quality, the generation of a digital elevation model, and orthorectification. The AVNIR-2 processor comprises radiometric correction, orthorectification, and atmospheric correction over land. Here, we present the methodologies applied during these processing steps as well as the results achieved using the processors.
\end{abstract}

Index Terms-Advanced Land Observation Satellite (ALOS), atmospheric correction (AC), AVNIR-2, deconvolution, digital elevation model (DEM) generation, geometric correction, PRISM.

\section{INTRODUCTION}

A COOPERATIVE agreement was signed between the Japanese Aerospace Exploration Agency (JAXA) and European Space Agency (ESA) to provide Advanced Land Observation Satellite (ALOS) data to European and African users. The Applied Remote Sensing Cluster (CAF) at the German Aerospace Center (DLR) has vast experience in the processing of high-resolution panchromatic and multispectral air- and spaceborne images. ESA granted a contract to DLR to develop prototype processors for the optical payload of ALOS, i.e., the PRISM and Advanced Visible and Near-Infrared Radiometer 2 (AVNIR-2) instruments. In this paper, we provide a survey over the processing chain as a whole and some of the processing steps in detail.

In addition to the microwave sensor Phased Array type L-band Synthetic Aperture Radar (PALSAR), ALOS carries two optical sensors, i.e., PRISM and AVNIR-2 [1]. The PRISM instrument has three panchromatic (0.52$0.77 \mu \mathrm{m}$ ) cameras (nadir, forward, and backward) to enable the acquisition of in-track triplet stereo images. This technique was first successfully demonstrated on a space mission with MOMS-2 [2]. The PRISM forward and backward telescopes are inclined $+24^{\circ}$ and $-24^{\circ}$ from nadir to realize a base-to-height

Manuscript received November 26, 2008; revised January 9, 2009. First published April 24, 2009; current version published November 25, 2009.

P. Schwind, M. Schneider, G. Palubinskas, T. Storch, and R. Müller are with the Remote Sensing Technology Institute, German Aerospace Center (DLR), 82234 Wessling, Germany (e-mail: peter.schwind@dlr.de; mathias. schneider@dlr.de; gintautas.palubinskas@dlr.de; tobias.storch@dlr.de; rupert. mueller@dlr.de).

R. Richter is with the Remote Sensing Data Center, German Aerospace Center (DLR), 82234 Wessling, Germany (e-mail: rudolf.richter@ dlr.de).

Color versions of one or more of the figures in this paper are available online at http://ieeexplore.iee.org.

Digital Object Identifier 10.1109/TGRS.2009.2015941 ratio of 1 . In this paper, we will only discuss the processing of the triplet mode with its $35-\mathrm{km}$ swath width and the $2.5-\mathrm{m}$ spatial resolution (nadir view), although PRISM has other modes of operation as well.

The second processor is developed for the AVNIR-2 payload. This instrument records four channels in the visible near infrared (VNIR): blue $(0.42-0.50 \mu \mathrm{m})$, green $(0.52-0.60 \mu \mathrm{m})$, red $(0.61-0.69 \mu \mathrm{m})$, and near infrared (near infrared (NIR): $0.76-0.89 \mu \mathrm{m})$. The spatial resolution is $10 \mathrm{~m}$ (at nadir), the swath width is $70 \mathrm{~km}$ (at nadir), and the across-track pointing capability is $\pm 44^{\circ}$.

In the following sections, an overlook of the processing chain is given, followed by a more detailed description of several processing steps of the processing chain. Finally, the results achieved using our processors are discussed and conclusions are drawn.

\section{ALOS PROCESSORS}

The design of the ALOS processing chains for PRISM and AVNIR-2 is based on the experience with fully automated and partially ISO 9001-2000 certified processing chains for airborne [3] and spaceborne [4] missions. Similar to these processor chains, the newly developed ALOS optical data processors include system and radiometric calibration, parametric geocoding, and atmospheric correction (AC).

Both processors are operated through a joint processing control system which allows for the choice of the input, processing, and output parameters either by a graphical user interface or by a command line tool. The JAXA Level 0 data serve as raw data together with the corresponding auxiliary information such as orbit and attitude data as well as the currently valid sensor model.

Fig. 1 shows which products can be generated by the AVNIR-2 processing (left) and the PRISM processing chain (right) as well as the processors' major tasks. These will now be briefly described, where L stands for Level.

The transcription processors for AVNIR-2 and PRISM (AVNIR-2_L1a and PRISM_L1a) derive the data from the different information streams. Radiometric, spectral, and geometric correction and calibration tables are computed and appended but not yet applied.

The systematic and radiometric correction processors for AVNIR-2 and PRISM (AVNIR-2_L1b and PRISM_L1b) correct the L1a products for known effects, e.g., odd-even and nonuniformity, and convert these data to physical top-ofatmosphere (TOA) radiance values. No resampling is applied in this task. For the PRISM data, an additional improvement of the 

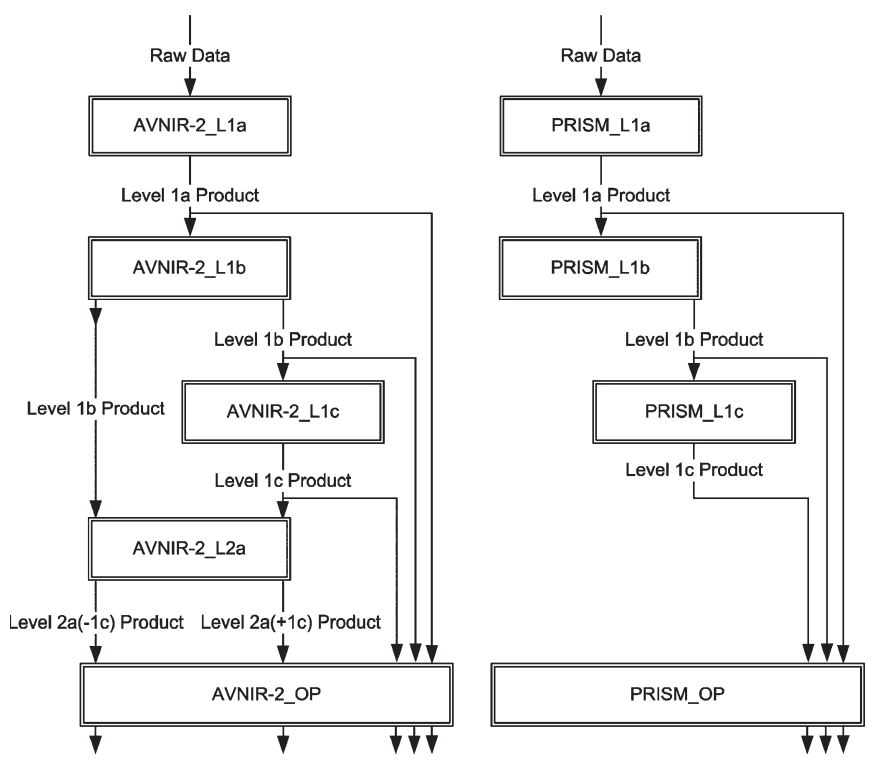

Fig. 1. Illustration of the processing chains for AVNIR-2 and PRISM.

image quality by a deconvolution step can be performed. These processors are described in [3] and even more detailed in [5].

The geometric correction processors for AVNIR-2 and PRISM (AVNIR-2_L1c and PRISM_L1c) generate orthoimages using the technique of direct georeferencing with an accurate digital elevation model (DEM). The line-of-sight (LoS) model uses onboard measurements of the star tracker systems for attitude determination, global positioning system (GPS) measurements for orbit determination (position and velocity), and sensor look direction vectors derived from laboratory and/or in-flight geometric calibration. Terrain displacements are taken into account by a global DEM which is also part of the processors (or given as input). A resampling, e.g., bicubic or bilinear, to a specified grid, e.g., according to Universal Transverse Mercator (UTM), is applied in this task. These processors are described in [4] and even more detailed in [6].

The AC processor for AVNIR-2 (AVNIR-2_L2a) first calculates cloud, haze, and water masks and a map of the aerosol optical thickness (AOT). Then, the surface reflectance image is computed based on a radiative transfer model using either the L1b or L1c product. Optionally, a haze removal can be applied. This processor is described in [6] and even more detailed in [7].

Finally, the output product processors for AVNIR-2 and PRISM (AVNIR-2_OP and PRISM_OP) finalize AVNIR-2 and PRISM products, namely, the image is converted to GeoTIFF and the metadata are formatted in Dimap [8], and generate processing information.

Even though not part of the PRISM prototype processing chain, an evaluation on orthoimage and DEM generation was performed. In a future operative processing chain, a DEM processor for PRISM (PRISM_DEM) would reconstruct the topology of the surface based on PRISM_L1b products of corresponding nadir, forward, and backward views. These images are radiometrically matched, and with information on orbit, attitude, and view direction, the surface elevation can be calculated. The evaluations for this processor are described in [4] and even more detailed in [6].

\section{PRISM Deconvolution Methods}

Remote sensing images are subject to distortions, such as motion blur, noise, atmospheric turbulences, etc. Image restoration techniques such as deconvolution and denoising can significantly improve the image quality and thus help in the visual interpretation and analysis/processing of images. Deconvolution is mathematically defined as the process reverting the convolution of a signal. In image processing, a complete reversion is usually not possible due to noise influence. Image deconvolution is, however, still applicable to compensate for undesired distortions to the image. To find the best deconvolution approach for PRISM images, two well-established methods, namely, Wiener and Richardson-Lucy (RL) deconvolution, as well as a more sophisticated approach, based on complex wavelet packets (complex wavelet packet automatic thresholding (COWPATH) [9]), were tested.

The convolution of an image $o$ with a point spread function (PSF) $h$ can be expressed as

$$
I(x, y)=O(x, y) H(x, y)
$$

where $I, O$, and $H$ are the Fourier transforms of $i, o$, and $h$, respectively. Based on this, in theory, deconvolution of an image can be described as

$$
O(x, y)=I(x, y) H(x, y)^{-1} .
$$

Unfortunately, such a simple inverse filter is not feasible in practice as the convolved image is usually influenced by noise, which is amplified by the inverse filter. Hence, most image restoration approaches differ in the way they deal with the influence of noise.

\section{A. Wiener Deconvolution}

As it is hard to obtain the original image $O$ due to the beforementioned noise influence, the Wiener filter, at least, tries to obtain the deconvolved image $\widehat{O}$ which has the minimum mean-square error with respect to the original image. Wiener deconvolution is defined as

$$
\widehat{O}(x, y)=H(x, y)^{-1}\left[\frac{|H(x, y)|^{2}}{|H(x, y)|^{2}+\frac{S_{n}(x, y)}{S_{o}(x, y)}}\right] I(x, y)
$$

where $S_{o}(x, y)$ and $S_{n}(x, y)$ are the power spectra of the original image and the noise, respectively.

\section{B. RL Deconvolution}

Unlike the other two algorithms presented here, the RL method uses an iterative approach to obtain the undistorted image. The resulting image is the one with the maximum probability to restore the blurred image when convolved with the PSF. The original RL implementation [10], [11]

$$
\widehat{O}_{i+1}(x, y)=\widehat{O}_{i}(x, y)\left[\overline{H(x, y)} \frac{I(x, y)}{H(x, y) \widehat{O}_{i}(x, y)}\right]
$$

only works for images influenced by Poisson-distributed noise. As remote sensing images usually suffer from additive 


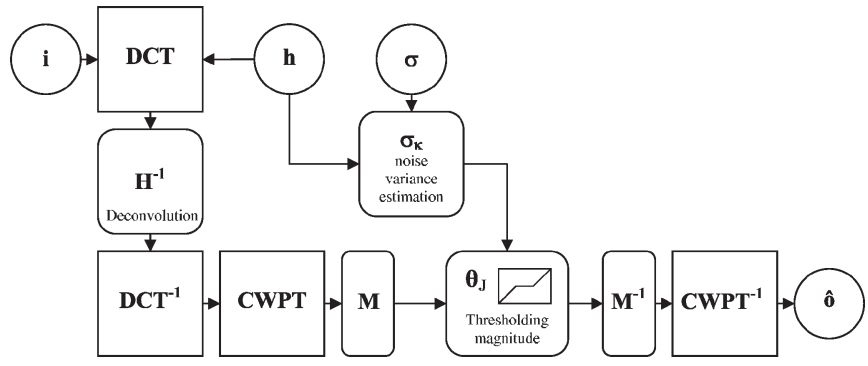

Fig. 2. COWPATH algorithm. Matrix $M$ transforms the wavelets from quadtree to complex representation and back [9].

Gaussian noise, a modified implementation, optimized for Gaussian noise [12], was used

$$
\widehat{O}_{i+1}(x, y)=\widehat{O}_{i}(x, y) \frac{I(x, y) \overline{H(x, y)}}{\left[H(x, y) \widehat{O}_{i}(x, y)\right] \overline{H(x, y)}} .
$$

It has already been shown that this modified version performs favorably compared to Wiener and regularized deconvolution [13] methods when dealing with satellite imagery [14].

\section{COWPATH}

The COWPATH algorithm (Fig. 2) can be split up into two main processing steps: deconvolution and denoising. First, the image is transformed using a discrete cosine transform. The transformed image is then divided by $H$, basically performing a simple inverse filter. As this step amplifies the noise, a complex wavelet packet thresholding algorithm is applied to the image. Wavelet-domain thresholding algorithms have been in use for some time in the signal processing community for noise reduction [15]. What differentiates complex wavelet packet thresholding from similar thresholding algorithms is that it is shift invariant and provides good directionality properties [16]. To obtain an efficient threshold for the denoising step, Jalobeanu et al. [9] first compute the noise variance $\sigma_{k}^{2}$ for each subband $k$ of the complex wavelet packet transform which is used to estimate an efficient threshold for each of the subbands for the final thresholding step. These authors suggest two different approaches for the coefficient thresholding step:

1) coefficient thresholding using numerically computed functions;

2) coefficient thresholding using a noninformative prior [17].

In this paper, the second alternative was chosen, as this approach showed slightly better results in the test cases presented by [9].

Even though COWPATH seems quite extensive compared to other deconvolution algorithms, the study in [9] showed that implementing the entire algorithm is possible with a linear time complexity.

The basis of an accurate image deconvolution is parameters describing the distortions of the image as precise as possible. Since these parameters [PSF, noise standard deviation, and signal-to-noise ratio (SNR)] were not available in this paper, they had to be estimated using available PRISM imagery (see [5] for details).

\section{Deconvolution Example}

Even though the approximate differences between a blurred and a reconstructed image are usually easy to spot to a human observer, comparison of images reconstructed using different settings or algorithms is not a trivial task. That is why, in addition to a visual interpretation, the three tested methods were also compared using two different metrics, namely, SNR and root mean squared error (rmse). To compare the different methods, test images were first convolved with a known PSF and Gaussian noise was added to the images. The images were then processed with the three deconvolution methods using the known PSF and noise standard deviation/SNR. Finally, the original and the reconstructed images were used to compute the SNR and the rmse [5]. Out of the three tested algorithms, the Wiener filter achieved the worst scores, while RL deconvolution outperformed the other algorithms after more than 16 iterations (for more detailed results, refer to [5]). It should be noted that a high SNR/low rmse does not always mean that an image is visually more appealing to a human observer.

Next, we present a visual comparison of the deconvolution performance using the estimated parameters extracted from PRISM imagery. The resulting deconvolved images can be seen in Fig. 3.

Using Wiener deconvolution seems to have the smallest impact on the visual appearance of the image [Fig. 3(a)]. The contrast of the image seems to have improved somewhat, but at the same time, some details of the image seem to have been blurred, showing that this method is a compromise between inverse filtering and noise smoothing. The image deconvolved using RL deconvolution [Fig. 3(c)] looks pretty similar to the previous image, except for the fact that the image was not smoothed as much. Finally, the image deconvolved using the COWPATH algorithm again improves the contrast of the image but, at the same time, seems to better preserve some structures of the image than RL deconvolution. By visual inspection, it looks like this method also handles the noise better than the other two tested algorithms.

A disadvantage of all three tested methods is that the JPEG artifacts present in PRISM imagery are amplified by the image reconstruction, since these algorithms were not designed with JPEG noise in mind. This is particularly noticeable in homogeneous areas (Fig. 3). Even though it is impossible to get rid of these artifacts completely, there are JPEG noise reduction algorithms available which could probably improve the image quality to some extent [18].

\section{PRISM Stereo EVAluation}

In addition to radiometric evaluations, geometric considerations have been performed regarding orthoimage and digital surface model (DSM) generation. In this chapter, results are presented for three test sites.

The first site is located in Catalonia near Barcelona (Spain). Two sets of radiometrically corrected triplet images of PRISM are available, acquired in October 2006, and processed by the ESA processor. In addition, five orthophotos for ground control point (GCP) and image control point measurements, as well as a DEM of the test site with 15-m resolution, were provided by the Institut Cartografic de Catalunya. Data for a second test site near Marseille (France) were recorded in 


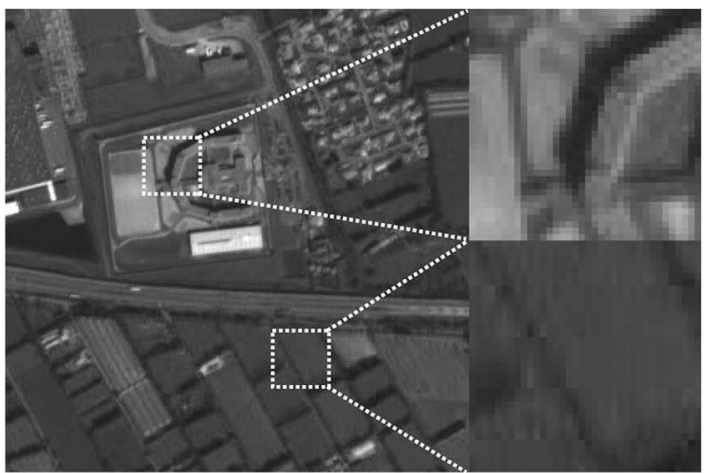

(a)

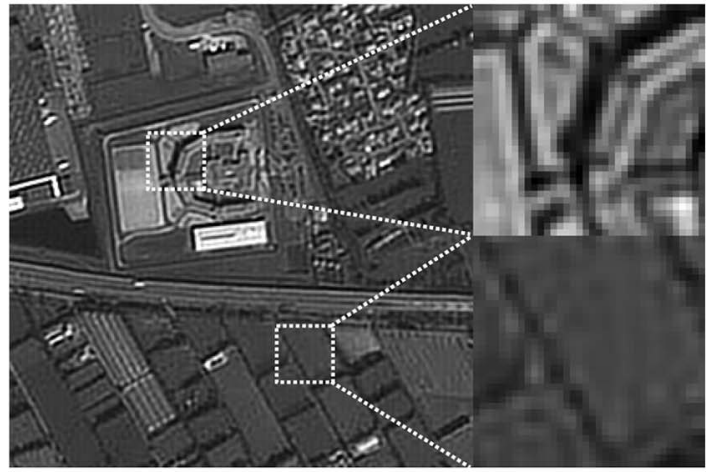

(b)

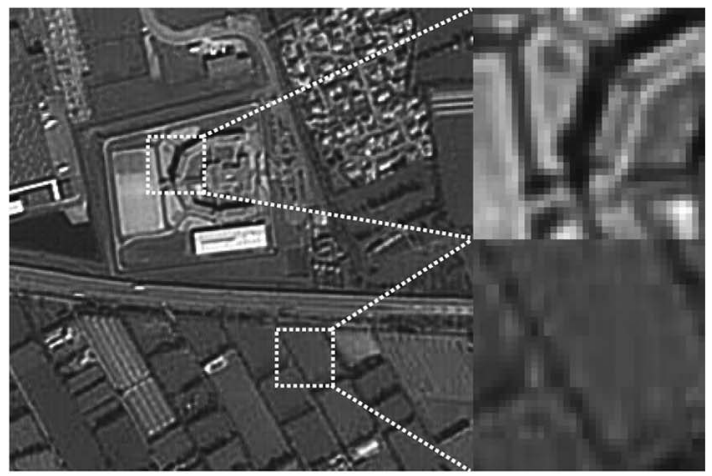

(c)

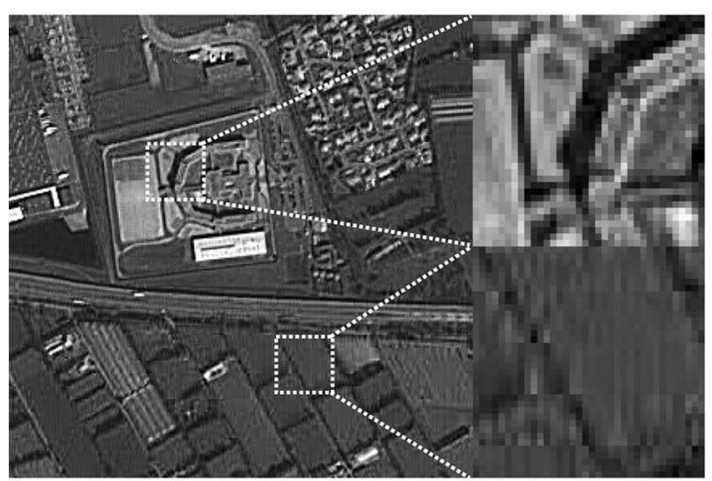

(d)

Fig. 3. Original image (a) deconvolved using (b) Wiener deconvolution, (c) RL deconvolution, and (d) the COWPATH algorithm.

March 2007 and provided to DLR by GAEL Consultant. Coordinates of six GCPs were provided, measured with GPS, as well as a DEM with 100-m resolution. This PRISM data set was processed at JAXA. The third data set is newer, so that,

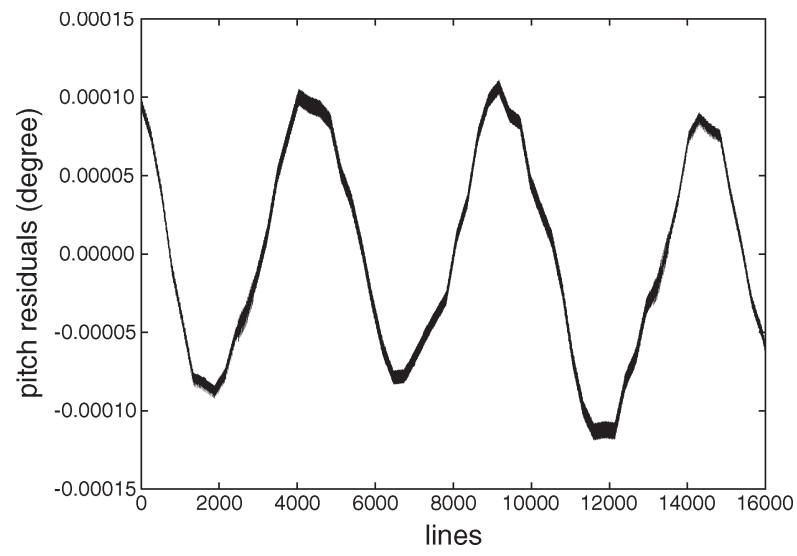

Fig. 4. Residuals of pitch angle after subtracting a second-order polynomial (nadir image of Catalonia).

hopefully, most of the parameters are improved. The third test site is located near Munich in Germany. Aside from the PRISM images, five orthophotos, as well as a Shuttle Radar Topography Mission DEM of the area, are available. The PRISM images were acquired in June 2007 and processed in September 2008 at ESA.

The exterior orientation consists of the roll, pitch, and yaw angles and the satellite position at the imaging time in Earth centered rotated (ECR) coordinates (per image line). The interior PRISM orientation is given in a table as view vector for each pixel (see [6] for details). GCPs were used to estimate the boresight angles.

\section{A. RPC Generation}

The first investigation concerns the generation of rational polynomial coefficients (RPCs) and the associated accuracy. For this purpose, a 3-D grid of control points is generated per scene, well distributed over the whole test image, using the exterior and interior orientation. The RPCs are then computed as described in [19]. To check the accuracy of the RPCs, coordinates of the control points were recalculated with the RPCs and compared to the original coordinates. For the older data sets (Spain, France), the residuals are smaller than one pixel; however, they may affect the quality of the DEM generation. Regarding the residual behavior in row direction, an oscillation with an amplitude of approximately one pixel can be found in the scenes. An examination of the attitude angles shows a linear behavior. However, if a second-order Legendre polynomial is employed as a trend estimate and if this trend is subtracted from the original values, an oscillation is clearly visible, e.g., in Fig. 4, which shows the pitch angle residuals for the nadir view of the Catalonia scene. A similar behavior can be found for the yaw angle. Residuals for the roll angle have a slightly smaller amplitude. However, when analyzing the newest data set (Germany), this oscillation is no longer visible. The absence of the oscillation in the newest data set might either be caused by the improved sensor model, or the oscillation might be caused by something that does not occur permanently. Maybe, it is caused by PALSAR working at the same time, or the oscillation may result from vibrations caused by satellite steering, etc. Similar oscillations are also known from other cases, e.g., MOMS-2P [20]. 


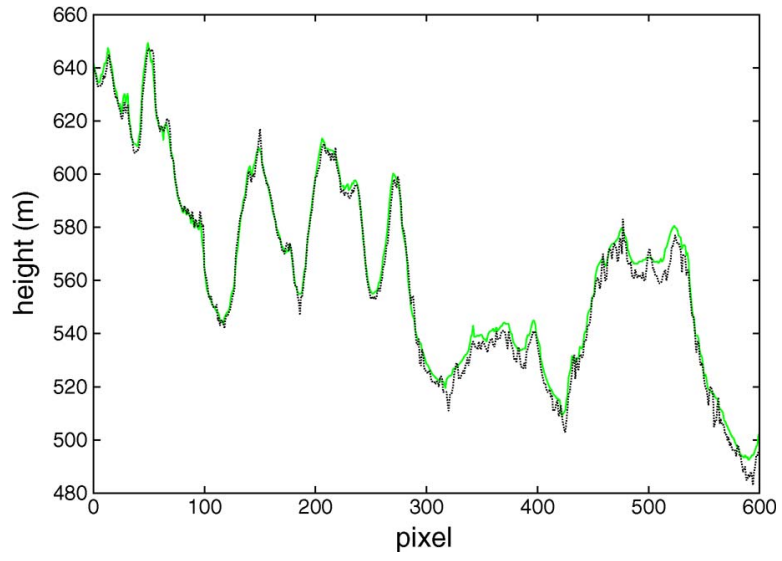

Fig. 5. Comparison of a profile in (black/dotted) PRISM DSM and (bright/solid) reference DEM in the northwestern part of the images. The profile is across track.

Due to the high spatial resolution of PRISM, these oscillation effects are not negligible as they are for SPOT. Oscillation amplitudes of up to one pixel might be tolerable for orthoimage generation; however, it will adversely affect the DEM generation from PRISM triplets. Since the reason for the oscillation is still unknown, this effect has to be examined further in the future. In case that the oscillation also occurs in newly processed data sets, the RPC-based approach is not fully suitable for PRISM imagery, since the effects cannot be handled by RPCs. Then, the rigorous model should be used. In case that the oscillation was eliminated by the improved parameters and does not occur any more, the RPC-based model can be used.

\section{B. DSM Generation}

An example of DSM generation is presented for the Catalonian test site using the RPC-based approach, knowing that the oscillation described in Section IV-A will occur. However, the resulting errors are small enough to be ignored for a general examination of PRISM DSM potential. Therefore, after matching of forward, nadir, and backward images, a forward intersection is computed for the tie points. Three and two ray points are used. A DSM is then interpolated from the resulting mass points. The DSM is then compared to the reference DEM provided by the Cartographic Institute of Catalonia. Fig. 5 shows a comparison of elevations in the northwestern part of the test site. The profiles show a very good correlation both in position and in height.

Due to the immense amount of points and the resulting size of the files, the processing is done in eight chips. When merging these chips to one DSM, differences may occur in the overlapping areas, particularly in areas where only few points are found, for example, in dense forests or in large agricultural areas. This effect worsens the overall statistics shown in Table I. Therefore, the statistics are also calculated for the northwestern DSM chip. There are some few outliers probably caused by mismatchings or interpolation artifacts; however, the mean difference is very small. In addition, the standard deviation is quite small, particularly when regarding the chip. The DSMs for the French and German test sites deliver similar results. The remaining differences result from typical DSM generation
TABLE I

STATISTICS ON DifFERENCE IMAGE BETWEEN PRISM DSM AND REFERENCE DEM For Catalonian TEST Site. Values Are Given in Meters

\begin{tabular}{lcc}
\hline & Overall & Chip 1 \\
\hline Min & -134.430 & -81.540 \\
Max & 277.740 & 93.040 \\
Mean & 0.936 & -0.231 \\
Std.-dev. & 10.679 & 3.964 \\
\hline
\end{tabular}

TABLE II

RMSE VALUES AT GCPS Before IMPROVEMENT OF ATTITUdE ANGLES in Data Sets From Spain (October 2006), France (March 2007), AND GERMANy (SEPTEMBER 2008)

\begin{tabular}{lccc}
\hline & & RMSEx [pixel] & RMSEy [pixel] \\
\hline Spain (25 GCP) & F & 8.187 & 7.929 \\
& N & 90.282 & 15.936 \\
& B & 38.763 & 15.988 \\
\hline France (6 GCP) & F & 23.405 & 9.525 \\
& N & 32.604 & 2.331 \\
& B & 4.927 & 7.168 \\
\hline Germany (33 GCP) & F & 4.787 & 3.246 \\
& N & 6.631 & 3.191 \\
& B & 2.712 & 1.071 \\
\hline
\end{tabular}

problems, such as the difficulty to match points in areas with a uniform texture, and are not PRISM-specific problems.

\section{Example of PRISM Orthoimage}

GCPs were measured in all images. The DLR-developed software ESTIMATE is used to estimate boresight angles. As an additional result, ESTIMATE returns the rmse values at the GCPs before the improvement. Table II shows the results for the different test regions. Due to the improved parameters, particularly in ancillary file 13, the location accuracy improved drastically. For the older data sets, the use of GCPs is inevitable, whereas for the newest data set-depending on the desired accuracy - an orthorectification or DEM generation without GCPs is possible. Fig. 6 shows an example of an orthoimage for the German test site.

\section{AVNIR-2 Orthoimage Generation}

The generation of orthoimages from AVNIR-2 data is based-as for the PRISM data - on the technique of direct georeferencing using the characterization of the image/pixel generation process [21], [22]: from the focal plane location of an instrument pixel given in the sensor's coordinate frame to the Earth surface location in terms of Earth-bound coordinate frames (e.g., an LTS local topocentric coordinate frame serves as model coordinate frame). The whole orthorectification process-realized by the DLR in-house generic orthorectification processor ORTHO [23] — starts with the establishment of the LoS model, which uses the measurements of the precision orbit data (state vectors, attitude, and timing information), the sensor mounting, and the sensor model (laboratory geometric calibration values and/or refinements by in-flight calibration). The ALOS precision orbit data contain time-tagged state vectors (satellite position and velocity) which are expressed in ECR frame with a sampling distance of $60 \mathrm{~s}$. For each image line, the state vectors are determined by Lagrange interpolation in order to preserve the expected position accuracy of about $2 \mathrm{~m}$. 


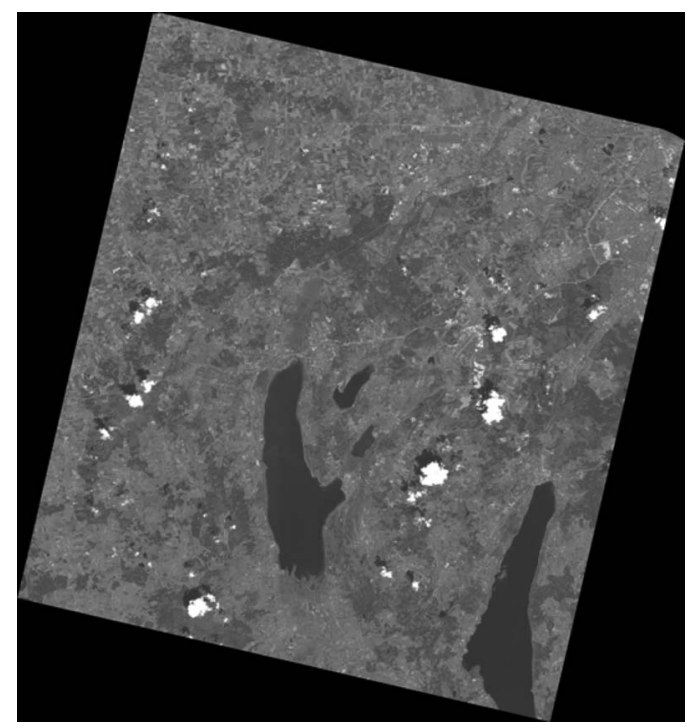

Fig. 6. Example of a PRISM orthoimage: German test site.

The satellite attitude data are given in unit quaternions with a sampling frequency of $10 \mathrm{~Hz}$ expressed in Earth centered inertial (ECI) frame.

The quaternions are transformed to Euler angles with specific rotation sequence (roll-pitch-yaw) and linearly interpolated for each image line. The ECI J2000 (ECI with reference year 2000) to ECR ITRF97 (ECR with datum International Terrestrial Reference Frame from 1997) transformation is performed using the Nutation/Precession matrix and the Polar Motion matrix provided in the ALOS auxiliary data with a sampling distance of $60 \mathrm{~s}$ and the Earth rotation accounting for the Greenwich apparent sidereal time with respect to a reference time and reference angle. The AVNIR-2 sensor model contains the relative alignment of the multispectral channels with respect to the master channel 3, the lens distortion parameters, and the tilt angle of the pointing mirror (up to $22^{\circ}$ across track resulting in an off-nadir view of $44^{\circ}$ ). Finally, the sensor mounting with respect to the Star Tracker coordinate frame (body frame) is taken into account. Because the mounting alignment strongly depends on temperature influences during orbit revolution, the orthorectification accuracy can be significantly improved by estimation of new mounting angles using GCPs.

The next orthorectification step determines the intersection between the LoS vector and the DEM by an iterative process, which results in a 3-D point in object space (LTS coordinates) for each image pixel. The reconstructed object points are finally transformed to map coordinates (e.g., UTM), where resampling (nearest neighbor, bilinear, and cubic convolution) leads to the orthoimage.

The AVNIR-2 CCD detector consists of 7100 elements in a staggered array layout with an odd-even along-track separation of about five-pixel sizes resulting in a footprint separation of 5-7 pixels depending on the tilt angle of up to $44^{\circ}$ (see Fig. 7). Therefore, prior to the geometric correction of the AVNIR-2 images, the odd and even parts have to be coregistered. The basic idea of coregistration is to establish a relationship between the odd and even parts in image space via object space and transform one subpart of the image (e.g., even part) to the gaps of the other part of the image (for example, odd).

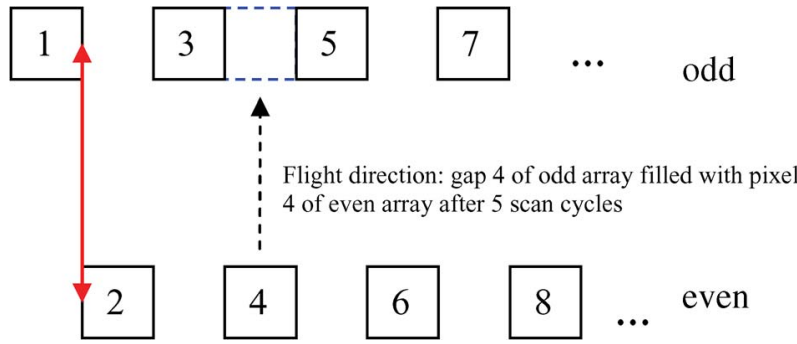

Fig. 7. AVNIR-2 staggered array layout with an odd-even along-track separation of five pixels. The gaps of the odd part of the array have to be filled with pixel values of the even part of the array in order to achieve a coregistered image.

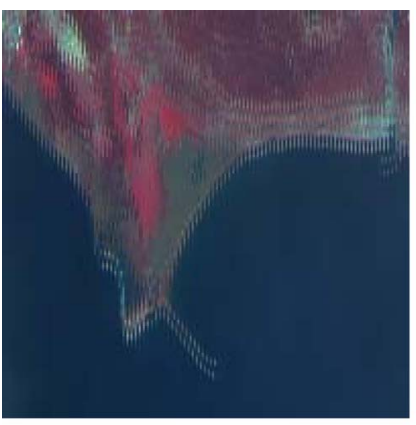

(a)

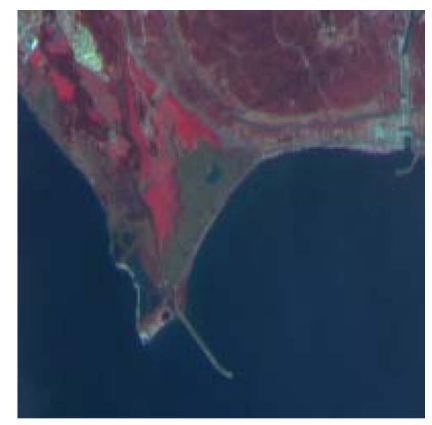

(b)
Fig. 8. Example of AVNIR-2 odd-even coregistration (false color image chip, $200 \times 200$ pixel).

It is assumed that proper yaw steering of the satellite has been performed during image acquisition in order to be able to fill the interspaces of the odd array correctly by the time-separated even pixels. The linear relation of the form

$$
f(X, Y)=a_{1}+a_{2} X+a_{3} Y+a_{4} X Y
$$

between object-space coordinates $(X, Y)$ and image-space coordinates $(u, v)$ is given for the odd and even pixels by

$$
\begin{array}{ll}
u_{\text {odd }}=f_{\text {odd }_{u}}(X, Y) & u_{\text {even }}=f_{\operatorname{even}_{u}}(X, Y) \\
v_{\text {odd }}=f_{\text {odd }_{v}}(X, Y) & v_{\text {even }}=f_{\text {even }_{v}}(X, Y)
\end{array}
$$

which directly leads to a linear relation between the odd part of the image and the even part of the image

$$
u_{\text {odd }}\left(u_{\text {even }}, v_{\text {even }}\right)=c_{1}+c_{2} u_{\text {even }}+c_{3} v_{\text {even }}+c_{4} u_{\text {even }} v_{\text {even }}
$$

$v_{\text {odd }}\left(u_{\text {even }}, v_{\text {even }}\right)=d_{1}+d_{2} u_{\text {even }}+d_{3} v_{\text {even }}+d_{4} u_{\text {even }} v_{\text {even }}$

which means that the gap pixels in the odd array can be filled with real pixels from the even array. Because this linear relationship is not valid for the whole scene, the image is subdivided into a grid of $100 \times 100$ pixels, where the linear relation holds with sufficient accuracies (empirical value with mapping accuracies of $<0.1$ pixel). The interspaces of the odd array are filled with linear interpolated even pixels (1-D interpolation in flight direction). An example is shown in Fig. 8. 


\section{AVNIR-2 AC}

The AC method is based on radiative transfer calculations with the MODTRAN4 code [24], [25]. A comprehensive set of calculations is performed for different atmospheric conditions, and solar and view geometries. Results are stored in a database of lookup tables (LUTs). These high-spectral-resolution LUTs are then resampled with the AVNIR-2 channel filter curves, and the AC can be conducted as described in [26] and [27]. The usual Lambertian assumption is employed for the surface reflectance law, and the standard rural (continental) aerosol model [25] is selected, because a reliable estimate of the aerosol type over land is not possible with a few VNIR channels. The influence of the atmospheric water vapor column is very small for the AVNIR-2 channels [7], and since a water vapor map cannot be derived from an AVNIR-2 scene, a typical seasonal/geographic value has to be taken. The same argument applies to the ozone column; again, a fixed value pertaining to the selected climatology of the MODTRAN standard atmospheres is used (e.g., midlatitude summer, winter, U.S. standard, and tropical).

Remaining input parameters to the $\mathrm{AC}$ are the acquisition date, solar zenith and azimuth angles, AVNIR-2 off-nadir pointing angle and azimuth, a DEM matched to the scene, and the orthorectified AVNIR-2 scene. In case of a mountainous terrain, the slope and aspect maps are calculated for a combined atmospheric/topographic correction before the atmospheric processor starts.

\section{A. Major Processing Steps}

The surface reflectance retrieval is the last step of any AC. The complete sequence of major processing steps of AC algorithms over land can be summarized as follows.

1) Masks are generated for land, water, haze over land, cloud, and saturated pixels.

2) The map of the AOT (at $550 \mathrm{~nm}$ ) is calculated.

3) An optional haze removal is performed.

4) The surface reflectance retrieval is conducted.

The first step of generating the masks is currently calculated with spectral threshold criteria. It is obvious that a unique characterization is not possible with only four VNIR bands; therefore, ambiguities and misclassifications might occur. Nevertheless, results so far indicate a satisfactory performance of the spectral masking algorithm. Future improvements are possible if spatial criteria (pattern analysis and neighborhood information) are included. As an example of the currently employed spectral criteria, the water mask is computed with

$$
\rho^{*}(\text { blue })<0.2 \quad \frac{d \rho^{*}(\lambda)}{d \lambda}<0
$$

where $\rho^{*}$ and "blue" indicate the TOA reflectance and the blue channel of AVNIR-2, respectively. The first part of (11) will include shallow water over bright sands in the water mask. This situation often occurs in coastal regions. The second part mimics the typical behavior of a negative TOA reflectance gradient in the blue-to-NIR region. It is essential to use the TOA reflectance and not the surface reflectance in this equation (see [7] for a detailed discussion of all spectral masking criteria).

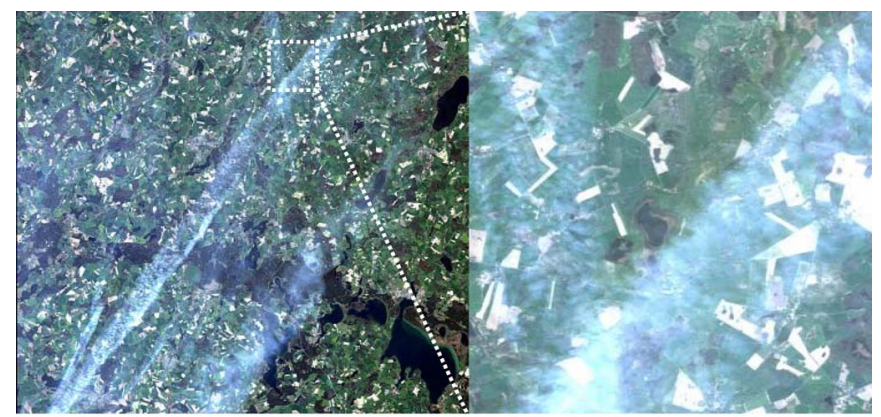

(a)

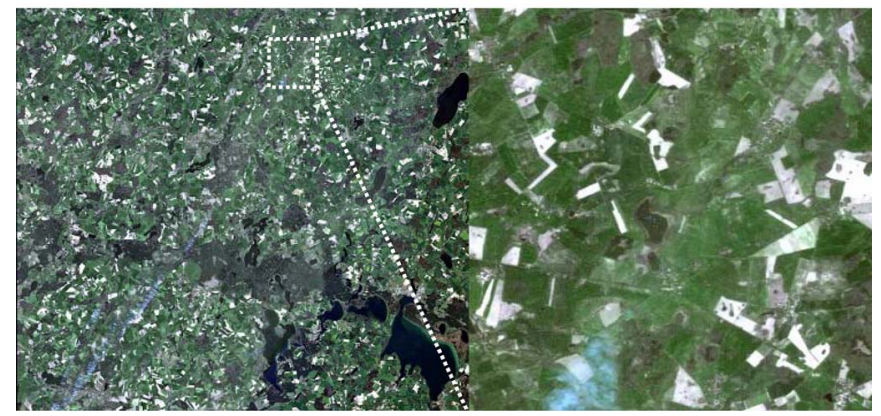

(b)

Fig. 9. AVNIR-2 scene of northern Germany (on April 16, 2007).

Next, a search for dark reference pixels is conducted in the clear part of the scene to determine the AOT at $550 \mathrm{~nm}$. Since AVNIR-2 lacks shortwave infrared bands in the 1.6- or $2.2-\mu \mathrm{m}$ regions, the dense dark vegetation algorithm of [28] cannot be applied. Therefore, another empirical method with a reduced accuracy has to be employed [29]. In addition, if the scene contains water bodies, the surface reflectance of water pixels in the NIR channel is checked: If negative reflectance pixels are encountered, then the AOT is iteratively decreased (visibility increased) until the surface reflectance is positive or the maximum number of iterations is reached. The AOT for nonreference pixels is set to the average value of the AOT value of the reference pixels; alternatively, a spatial interpolation can be applied. If the scene contains no reference and no water pixels (e.g., desert regions), the processing is done with a constant visibility of $23 \mathrm{~km}$. Since desert regions usually consist of sand or soil with high reflectance values $(0.20-0.50$ reflectance units), the sensitivity with respect to a wrong visibility estimate is rather low: for some example desert scenes in Libya, surface reflectance values changed less than 0.01 units for a visibility variation from 15 to $50 \mathrm{~km}$.

For a hazy scene, an optional haze removal follows [30], [7]. It works on the orthorectified digital number (DN) image and converts each "hazy" DN pixel into a "de-hazed" DN pixel. After this "de-hazing," the scene is considered as "clear" (except for cloud pixels, of course) and the surface reflectance retrieval based on the AOT map is conducted.

The final surface reflectance retrieval is performed iteratively to account for the adjacency effect [26], [27].

\section{B. Example Scene With Haze}

Fig. 9(a) shows an AVNIR-2 scene of northern Germany recorded on April 16, 2007. The scene contains thin and thick 


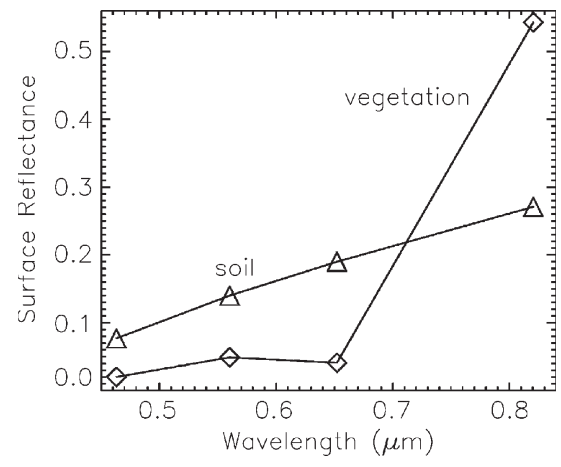

Fig. 10. Reflectance spectra of soil and vegetation from hazy area.

haze over different areas. The solar zenith and azimuth angles are $46.8^{\circ}$ and $150.6^{\circ}$, respectively. The instrument pointing angle is $41.5^{\circ}$ with a $98.1^{\circ}$ azimuth. Fig. 9(b) shows the result after applying the haze removal and surface reflectance retrieval. The visual impression demonstrates a successful removal of haze effects, even in areas with moderately thick haze (compare the zoomed views in Fig. 9).

Fig. 10 shows a soil and a vegetation spectrum from a hazy area. The spectral shape and reflectance values are consistent and within the expected range. They are also close to spectra from neighborhood areas that are not affected by haze. However, a comparison with spectroradiometric field measurements is not yet available but should be planned for the future.

\section{CONCLUSiON}

Two processors for PRISM and AVNIR-2 imagery have been developed for ESA. They comprise the conversion from Level 0 to Level 1 products (systematic and radiometric corrections), an optional deconvolution, and geometric and atmospheric (only AVNIR-2) corrections. Concerning the tested deconvolution methods, the modified RL deconvolution outperforms the Wiener and COWPATH algorithms based on the achieved SNR and rmse. However, a visual analysis ranks the COWPATH first, followed by RL and Wiener.

The RPC-based approach on PRISM images reveals oscillations of up to one pixel in older data sets. While this might be tolerable for orthoimage products in most applications, it will adversely affect the DEM generation. In the newest data set, the oscillation no longer exists, maybe due to the improved sensor model parameters. Another possible reason for the oscillation might be vibrations caused by some satellite instrument that is not working permanently, e.g., PALSAR. In case that the oscillation has permanently vanished, an RPC-based approach is applicable. If not, we recommend the use of a rigorous approach rather than an RPC-based approach. This has to be examined further in the future. The georeferencing results improved when using newly processed data with updated sensor model parameters. Tests showed that, while, for older data sets, GCPs have to be used to estimate boresight angles, for the newer data set, orthorectification and DSM generation are possible without GCPs with an accuracy of 10-15 m. Comparison of PRISM DSM and reference DTM reveals a very high quality of the PRISM DSM.

Just as for PRISM, the AVNIR-2 images are orthorectified using a physical model based on direct georeferencing tech- niques. Prior to the geometric correction, the odd and even parts of the AVNIR-2 image are coregistered, making use of the precise yaw steering of the satellite and the fact that the odd and even pixels are linearly related. To verify the accuracy of this approach, a more detailed evaluation will have to be conducted in the future.

The atmospheric processor of AVNIR-2 imagery produces three output images: 1) a map containing the distribution of haze, cloud, and water in a scene; 2) a map of the AOT at $550 \mathrm{~nm}$; and 3) the surface reflectance in four VNIR channels. Test scenes with and without haze were successfully processed. Visual quality checks and consistency checks concerning the derived surface reflectance signatures were conducted. A comparison with ground-based reflectance measurements is planned in the future.

\section{REFERENCES}

[1] T. Tadono, M. Shimada, M. Watanabe, and A. Rosenqvist, "Overview of ALOS research and science program," Proc. SPIE, vol. 5570, pp. 10-21, 2004.

[2] J. Bodechtel, Q. Lei, G. Loercher, H. Kaufmann, M. Berger, and B. Koch, "MOMS-02/D2: First results and future applications," Int. J. Remote Sens., vol. 15, no. 13, pp. 2513-2520, Sep. 1994.

[3] M. Habermeyer, A. Müller, S. Holzwarth, R. Richter, R. Müller, M. Bachmann, K.-H. Seitz, P. Seifert, and P. Strobl, "Implementation of the automatic processing chain for ARES," in Proc. EARSeL Workshop Imaging Spectroscopy, Warsaw, Poland, 2005, pp. 67-75.

[4] T. Storch, A. de Miguel, R. Müller, A. Müller, A. Neumann, T. Walzel, M. Bachmann, G. Palubinskas, M. Lehner, R. Richter, E. Borg, B. Fichtelmann, T. Heege, M. Schroeder, and P. Reinartz, "The future spaceborne hyperspectral imager EnMAP: Its calibration, validation, and processing chain," in Proc. 21st Congr. Int. Soc. Photogramm. Remote Sens., Beijing, China, 2008, pp. 1265-1270.

[5] P. Schwind, G. Palubinskas, T. Storch, and R. Müller, "Evaluation of deconvolution methods for PRISM images," in Proc. ALOS PI Symp., Rhodes, Greece, 2008.

[6] M. Schneider, M. Lehner, R. Müller, and P. Reinartz, "Stereo evaluation of ALOS/PRISM data on ESA-AO test sites: First DLR results," in Proc. ALOS PI Symp., Rhodes, Greece, 2008.

[7] R. Richter, "Improvements in atmospheric correction for multispectral VNIR imagery," Sensors, vol. 8, pp. 6999-7011, 2008.

[8] Complete Documentation of the New Format Called DIMAP. [Online]. Available: www.spotimage.fr/dimap.html

[9] A. Jalobeanu, L. Blanc-Fraud, and J. Zerubia, "Satellite image deconvolution using complex wavelet packets," in Proc. Int. Conf. Image Process., Vancouver, BC, Canada, 2000, vol. 3, pp. 809-812.

[10] W. H. Richardson, "Bayesian-based iterative method of image restoration," J. Opt. Soc. Amer., vol. 62, no. 1, pp. 55-59, Jan. 1972.

[11] L. B. Lucy, "An iterative technique for the rectification of observed distributions," Astron. J., vol. 79, p. 745, Jun. 1974.

[12] M. Pruksch and F. Fleischmann, "Positive iterative deconvolution in comparison to Richardson-Lucy like algorithms," in Proc. Astron. Data Anal. Softw. Syst. VII, 1998, vol. 145, p. 496.

[13] J. Biemond, R. L. Lagendijk, and R. M. Mersereau, "Iterative methods for image deblurring," Proc. IEEE, vol. 78, no. 5, pp. 856-882, May 1990.

[14] T. Bretschneider, "On the deconvolution of satellite imagery," in Proc. Geosci. Remote Sens. Symp., 2002, vol. 4, pp. 2450-2452.

[15] D. L. Donoho, "De-noising by soft-thresholding," IEEE Trans. Inf. The ory, vol. 41, no. 3, pp. 613-627, May 1995.

[16] N. G. Kingsbury, "The dual tree complex wavelet transform: A new efficient tool for image restoration and enhancement," in Proc. Eur. Signal Process. Conf., 1998, pp. 319-322.

[17] J. Bernardo and A. Smith, Bayesian Theory. New York: Wiley, 1994.

[18] I. Kamiya and G. Saito, "Reduction of JPEG and other noise for ALOS PRISM image," in Proc. 28th Asian Conf. Remote Sens., Kuala Lumpur, Malaysia, 2007.

[19] M. Lehner, R. Müller, and P. Reinartz, "Stereo evaluation of Cartosat-1 data for French and Catalonian test sites," in Proc. ISPRS Workshop High Resolution Earth Imaging Geospatial Inf., Hannover, Germany, May 29-Jun. 12007. 
[20] M. Lehner and R. Müller, "Quality check of MOMS-2P ortho-images of semi-arid landscapes," in Proc. ISPRS Workshop High Resolution Mapping Space, Hannover, Germany, 2003.

[21] ALOS/PRISM \& AVNIR-2 Level 1 Data Processing Algorithms, Oct. 2006. Revision J.

[22] Format Description of the ALOS Ancillary Information Files, Sep. 2005. Revision A-8.

[23] R. Müller, M. Lehner, P. Reinartz, and M. Schroeder, "Evaluation of spaceborne and airborne line scanner images using a generic ortho image processor," in Proc. ISPRS High Resolution Earth Imaging Geospatial Inf., C. Heipke, K. Jacobsen, and M. Gerke, Eds, Hannover, Germany, May 17-20, 2005, vol. XXXVI.

[24] A. Berk, L. S. Bernstein, G. P. Anderson, P. K. Acharya, D. C. Robertson, J. H. Chetwynd, and S. M. Adler-Golden, "MODTRAN cloud and multiple scattering upgrades with application to AVIRIS," Remote Sens. Environ., vol. 65, no. 3, pp. 367-375, Sep. 1998.

[25] A. Berk, G. P. Anderson, P. K. Acharya, M. L. Hoke, J. H. Chetwynd, L. S. Bernstein, E. P. Shettle, M. W. Matthew, and S. M. Adler-Golden, MODTRAN4 Version 3 Revision 1 User's Manual. Hanscom AFB, MA: Air Force Res. Lab, 2003.

[26] R. Richter, "A spatially adaptive fast atmospheric correction algorithm," Int. J. Remote Sens., vol. 17, no. 6, pp. 1201-1214, 1996.

[27] R. Richter, "Correction of satellite imagery over mountainous terrain," Appl. Opt., vol. 37, no. 18, pp. 4004-4015, Jun. 1998.

[28] Y. J. Kaufman, A. E. Wald, L. A. Remer, B.-C. Gao, R.-R. Li, and L. Flynn, "The MODIS $2.1 \mu \mathrm{m}$ channel-correlation with visible reflectance for use in remote sensing of aerosol," IEEE Trans. Geosci. Remote Sens., vol. 35, no. 5, pp. 1286-1298, Sep. 1997.

[29] R. Richter, D. Schläpfer, and A. Müller, "An automatic atmospheric correction algorithm for visible/NIR imagery," Int. J. Remote Sens., vol. 27. no. 9/10, pp. 2077-2085, 2006.

[30] Y. Zhang, G. Guindon, and J. Cihlar, "An image transform to characterize and compensate for spatial variations in thin cloud contamination of Landsat images," Remote Sens. Environ., vol. 82, no. 2, pp. 173-187, Oct. 2002.

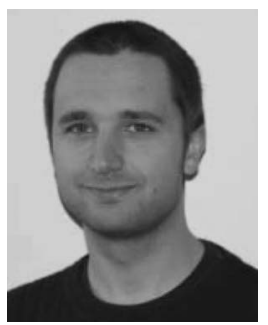

Peter Schwind received the Diploma [Dipl.-Inf. $(\mathrm{FH})]$ degree in computer science from the University of Applied Sciences, Landshut, Germany, in 2008.

Since then, he has been with the Photogrammetry and Image Analysis Unit, Remote Sensing Technology Institute, German Aerospace Center (DLR), Wessling, Germany. He is a member of the team responsible for the development of a "Prototype Processor for ALOS Optical Data" for the European Space Agency. His research interests include remote sensing, image registration, and deconvolution.

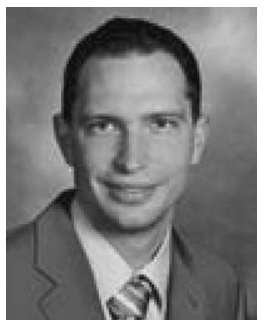

Mathias Schneider received the Dipl.-Ing. degree in geodesy and geoinformatics from the University of Stuttgart, Stuttgart, Germany, in 2004.

Since 2006, he has been with the "Matching and 3-D" group, Remote Sensing Technology Institute, German Aerospace Center (DLR), Wessling, Germany. He is working on a feasibility study in the frame of the GITEWS project. During the last year, he was also working on the geometric processing of ALOS imagery. Currently, he is a WP Manager of the Level 2A Processor of the German hyperspectral satellite mission EnMAP and Project Manager of the ESA-project Urban Atlas.

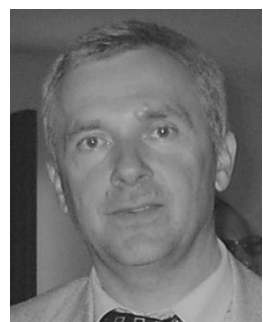

Gintautas Palubinskas received the M.S. degree in mathematics from Vilnius University, Vilnius, Lithuania, in 1981, and the Ph.D. degree in mathematics from the Institute of Mathematics and Informatics (IMI), Vilnius, in 1991. His doctoral dissertation was on spatial image recognition.

From 1981 to 1997, he was a Research Scientist with IMI. From 1993 to 1997, he was a Visiting Research Scientist with the German Remote Sensing Data Center, German Aerospace Center (DLR), Wessling, Germany; the Department of Geography, Swansea University, Wales, U.K.; the Institute of Navigation, Stuttgart University, Stuttgart, Germany; and Max-Planck-Institute of Cognitive Neuroscience, Leipzig, Germany. Since 1997, he has been a Research Scientist with the Remote Sensing Technology Institute, DLR. His current research interests include image processing, change detection, data fusion, and traffic monitoring for optical remote sensing applications.

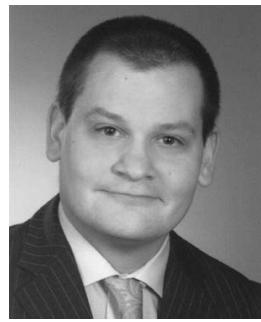

Tobias Storch received the Diploma (Dipl.-Inform.) and Doctorate (Dr. rer. nat.) degrees in computer science from the University of Dortmund, Dortmund, Germany, in 2003 and 2007, respectively.

As a Junior Researcher with the Remote Sensing Technology Institute, German Aerospace Center (DLR), Wessling, Germany, he conducts system engineering of fully automatic processing chains, in particular for optical remote sensing instruments of satellites. His current work focuses on ground segment engineering for the Environmental Mapping and Analysis Program mission, a future spaceborne hyperspectral imager.

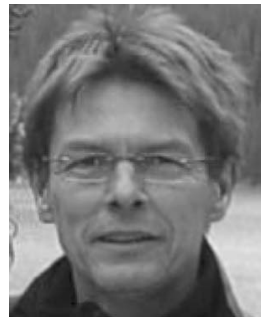

Rupert Müller received the Dipl.-Phys. degree from the Ludwig Maximilians University of Munich, Munich, Germany, in 1985.

$\mathrm{He}$ is currently a Team Leader of the "Processors and Traffic Monitoring" group, Remote Sensing Technology Institute, German Aerospace Center (DLR), Wessling, Germany, and is responsible for the Environmental Mapping and Analysis Program processing, calibration, and validation part within the ground segment as well as several European Space Agency projects like "Prototype Processor for ALOS Optical Data." His main research interests include photogrammetric evaluation of spaceborne satellite data and digital image processing.

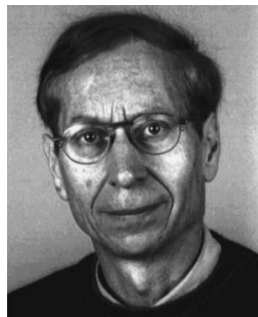

Rudolf Richter received the M.Sc. degree in physics from the Technical University of Munich, Munich, Germany, in 1973, and the Ph.D. (Dr. Ing.) degree in engineering from the Technical University of Dresden, Dresden, Germany, in 1991.

$\mathrm{He}$ is currently a Senior Scientist with the Remote Sensing Data Center, German Aerospace Center (DLR), Wessling, Germany, where he is conducting concept development, modeling, and simulation of airborne/spaceborne hyperspectral instruments. It involves advanced techniques and interaction with scientists associated with theory and remote sensing experiments. He developed the ATCOR model, one of the standard codes for atmospheric and topographic correction of multi-/hyperspectral imagery used at universities and research laboratories. His current work focuses on the design of fully automatic processing chains for the evaluation of remotely sensed optical data from the visible to the thermal spectral region. 